\title{
Article
}

\section{Land-cover Classification of Ehime Prefecture, Japan using Airborne Laser Altimetry}

\author{
Yoshiko Maeda*1, Hayato Tsuzuki*1, Ross Nelson ${ }^{* 2}$ and Tatsuo Sweda ${ }^{* 1}$
}

\begin{abstract}
Airborne laser profiling of mainland Ehime Prefecture, Japan was conducted to develop an entirely new method of land-cover classification, partly in preparation for the post-Kyoto national carbon budget accounting, and partly for correction of the existing government land-use statistics, which should constitute the very basis of the impending national carbon budget accounting of the Kyoto Protocol. The altimetry data was obtained by using NASA's Portable Airborne Laser System (PALS) along 23 parallel flight lines $4 \mathrm{~km}$ apart from each other covering the whole mainland portion of the prefecture. Based on the resulting surface profile representing topography and structures on the ground with some reference to laser return intensity and nadir video images, land cover along the flight line was classified into "forest", "farmland", "residential and urban", and "others" using PALSA (PALS Analyzer), a software developed for this particular purpose. This line evaluation was then developed into area statistics by simply multiplying by the distance between the flight lines, i.e. $4 \mathrm{~km}$. The resultant land-cover estimates not only differed from the existing government statistics as much as the latter does within itself, but also helped to identify the causes and sources of discrepancy quantitatively. Thus it was concluded that coordinated use of this new method with the existing system of land-use statistics would improve the overall credibility of the landuse/land-cover statistics of the prefecture and the nation.
\end{abstract}

Keywords: airborne laser altimetry, land-cover classification, Ehime Prefecture, discrepancy with government statistics, abandoned farmland

\section{INTRODUCTION}

As one of its prerequisites for national carbon budget accounting, the Kyoto Protocol demands accurate and consistent land-cover/land-use statistics from the Annex I countries. This is particularly important for a country like Japan which intends to utilize its forests as carbon sink to offset emission from other sources. However, as far as the existing land-use statistics are concerned, Japan does not seem to be capable of coping with this requirement of consistency due to contradicting figures among the statistics from different ministries, departments and agencies. Some of the discrepancies are identifiable as arising from difference in the definition

Corresponding author: Yoshiko Maeda

${ }^{* 1}$ Faculty of Agriculture, Ehime University, 3-5-7,

Tarumi, Matsuyama, Ehime 790-8566 Japan

*1 923/Biospheric Sciences Branch, NASA-Goddard

Space Flight Center, Greenbelt, MD, USA of land-use categories. However, they still leave considerable discrepancies, the source of which is either unidentifiable or can only be guessed at best, degrading the confidence in all the statistics involved.

As recommended by the Intergovernmental Panel on Climate Change (IPCC, 2003), the best tool for correcting such discrepancies of or renewing the existing statistics is remote sensing due to its capabilities of covering large areas to the remotest corners and of providing spatially explicit information repeatedly. Most probably being too new and too advanced, the airborne laser altimetry is not included in the IPCC list of means of remote sensing, but it can provide much better defined and more precise land classification than the conventional means of remote sensing as NELSON et al. (2003) has demonstrated for the state of Delaware, U.S.A. The present work was conducted to develop a new method of land classification for Japan using airborne laser altimetry for more consistent and accurate statistics in response to the impending Kyoto Protocol carbon budget reporting as well as in preparation for the post-Kyoto greenhouse gas inventorying. To be reasonably practical for countrywide application in not 
too distant future, Ehime Prefecture, one of the 47 prefectures of Japan, was chosen as the test area. The completely independent land-cover statistics thus created by airborne laser altimetry alone was compared with the existing government figures.

\section{EXISTING GOVERNMENT LAND-USE STATISTICS}

The two most comprehensive land-use statistics on Ehime Prefecture available today are the one on the Land Use Control bacK-up sYstem (LUCKY) from the Ministry of Land (LAND and Water BurEau, Ministry of LAND) and another from the prefectural statistics office (EHIME PrEFEcture STATISTICAL OfFICE (EPSO), 2005). The former is accompanied with a map while the latter is not. Of the numerous discrepancies between the two, the first to be mentioned is the $3.8 \%$ difference in total land area of the prefecture, i.e. $5,677 \mathrm{~km}^{2}$ by LUCKY against $5,247 \mathrm{~km}^{2}$ by Epso. The exact agreement of the former with the figure from the most authoritative Geographical Survey Institute (GSI) does not necessarily mean its accuracy since the statistical discrepancies are adjusted for the total land area from the GSI.

The discrepancies between the national and prefectural statistics arise partly from the difference in definition of landuse categories and partly from accuracy in data collection and compilation. As shown in Fig. 1 categorization of "Road", "Water" and "Rangeland" is explicitly different between the two. Implicit but more significant difference in definition exists in handling of agricultural land and forest reserve. The former is the area actually cultivated in the national statistics, while it is the area legally registered as such in the prefectural statistics. The latter is as a part of "Forest" in the national statistics, but it is a part of "Others" in the prefectural statistics.

These discrepancies are difficult to be resolved due to rather unaccountable difference in some of the actual figures, which in turn distort the entire proportion of the statistics. A typical example is "Forest", which appears $21.5 \%$ more in national statistics than in the prefectural. A small part of this disagreement can be accounted for by the difference in

\begin{tabular}{|c|c|c|c|}
\hline Prefectural (PSO) & \multicolumn{3}{|c|}{ National (LUCKY) } \\
\hline \multirow{2}{*}{ Forest } & \multicolumn{3}{|c|}{ Forest } \\
\hline & National fores & & ate forest \\
\hline Paddy & \multicolumn{3}{|c|}{ Farmland } \\
\hline Field & Paddy & & Rangeland \\
\hline \multirow[b]{2}{*}{ Urban and Built-up } & \multicolumn{3}{|c|}{ Urban and Built-up } \\
\hline & \begin{tabular}{l|l} 
Residential \\
$\mathrm{r}$
\end{tabular} & $\begin{array}{l}\text { Other } \\
\text { residential }\end{array}$ & Industrial \\
\hline Marsh & Water & & Canal \\
\hline Range- and Wasteland & \multicolumn{3}{|c|}{ Wasteland } \\
\hline \multirow{3}{*}{ Others } & \multicolumn{3}{|c|}{ Road } \\
\hline & Forest & tural & General \\
\hline & \multicolumn{3}{|c|}{ Others } \\
\hline
\end{tabular}

Fig. 1 Existing land-use classification of Ehime definition mentioned above, but the rest is beyond any other explanation than by what has been traditionally called "the stretched measure", which results in smaller figures than what really is. This was rather common when the modern land registration system was introduced a century ago partly due to under declaration of land area on the side of owners to evade tax, and partly due to lack of exact and efficient means of surveying on the side of government. The correction seems to have been almost completed by today for residential and agricultural lands due to their limited size and accessibility, but the measure seems still stretched for forests due to their vastness and inaccessibility. Relatively small difference between the national and prefectural statistics in the total land area as compared with that in "Forest" area, indicates possible distortion in the areas of other land uses.

\section{AIRBORNE LASER PROFILING OF EHIME PREFECTURE}

The airborne laser altimetry was flown in September 2005 in cooperation with the NASA using its Portable Airborne Laser System (PAIS) onboard a Bell Jet Ranger domestically hired from Akagi Helicopter Co. By this mission, the entire Ehime Prefecture excluding the islands off the mainland was covered with a total of 23 straight line transects $4 \mathrm{~km}$ apart from each other as shown in Fig. 2. By eliminating overlaps and approach flights to the transects, the total length of the flight lines used for land-cover classification and biomass estimation was $1,358 \mathrm{~km}$. PALS is a nadir ranging system with laser beam emission frequency of $2,000 \mathrm{~Hz}$, but only every five ranging data was retrieved along with return intensity resulting in a recording frequency of $400 \mathrm{~Hz}$. Nadir video picture was also taken for reference in later analysis.

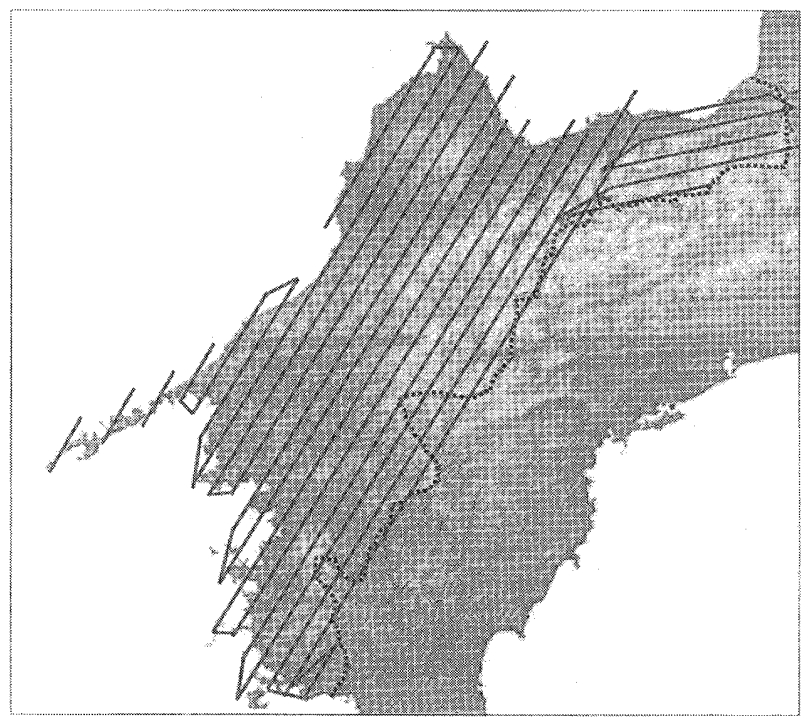

Fig. 2 Airborne laser altimetry Flight passage 


\section{CLASSIFICATION SOFTWARE}

A software named PALSA (PALS Analyzer) was developed for convenience and ease of land-cover classification with the PALS data. It displays surface profile and laser return intensity graphically along with video movie in coordination with each other on the computer screen. Classification has to be done manually by the operator by delimiting a continuous portion of the same land cover and specifying its type. Then the segment changes its color according to the prescribed landcover color code while the corresponding original altimetry records are flagged behind the screen according to the prescribed flag code for later statistical processing.

\section{LAND-COVER CLASSIFICATION}

With altimetry and return intensity recorded for every $17 \mathrm{~cm}$ along the flight track, detailed land-cover classification is possible with the PALS data. However, in the present analysis only four major categories were distinguished, i.e.: "Forest" where trees are growing including newly harvested areas where continued forestry use is obvious; "Agricultural" which includes paddy, cropland and orchard; "Residential and Urban" which cover all the buildup areas including industrial areas, airports, parking lots etc.; and "Others" which include all the other areas as golf courses not covered by the preceding categories. Roads were included in the category surrounding them. So were inland water surfaces since their extent is more or less negligible in mountainous Ehime Prefecture.

Classification was conducted in two steps with transect length of $20 \mathrm{~m}$ as a yardstick, i.e. when a given land cover continued for more than $20 \mathrm{~m}$ it was classified as such, but otherwise it was temporarily classified as "Mosaic" for more detailed classification later on. Theoretically, there are 11 ways of combination in choosing two or more cover categories out of the four major categories, but the actual mosaics encountered were only three kinds, i.e. Forest + Residential, Forest + Agriculture, and Agriculture + Residential. Being relatively little, the first two mosaic transects were simply reclassified. In the last category, however, only the sample sections of $2.3 \mathrm{~km}$ were reclassified into detail and the resulting proportion was applied to the remaining $19.1 \mathrm{~km}$ of mosaic segments. The cover classification results along the flight line were then converted into area statistics by simple rectangular approximation, i.e., by multiplying the flight-line length of a given cover category by the distance of $4 \mathrm{~km}$ between the flight lines.

\section{RESULTS AND DISCUSSION}

In spite of this seemingly very rough method of conversion into area, the total land area of the prefecture turned out to be $5,435 \mathrm{~km}^{2}$, which is only $0.4 \%$ less than the GSI

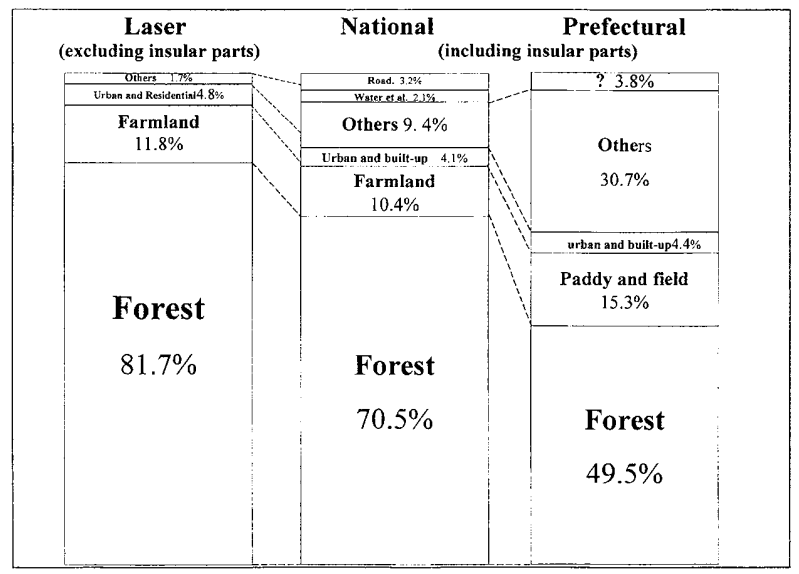

Fig. 3 Result of airborne laser altimetry land-cover classification as compared with national and prefecture statistics. (The areas of bar graph are not exactly to the percentage figures given due to space limitation)

figure of $5,455 \mathrm{~km}^{2}$. Considering the fact that the GSI figure for the mainland Ehime was obtained as the grand prefecture total less the area of islands over $1 \mathrm{~km}^{2}$, the actual discrepancy could be even smaller. On the other hand as shown in Fig. 3, the laser-based cover classification looks as contradicting with the existing statistics as the latter is within. However, the following detailed analysis leads to some consistency among the three estimates of land-cover/land-use classification.

Firstly, of the discrepancies in "Agricultural" land areas, the one between the laser estimate and the national statistics can be best explained by the fallow area, which amounts to $1 \%$ of the prefectural land area according to the 2005 Agroforestry Census (Ehime Prefecture Statistical Office, 2005). Unable to distinguish between them, the laser estimate of "Farmland" (11.8\%) may well includes the fallows in addition to the actually cultivated "Farmland" (10.4\%) given by the national statistics, to result in the remaining discrepancy of only $0.4 \%$. The further discrepancy of $3.5 \%$ with the "Paddy and Field" area (15.3\%) of the prefectural statistics can be best accounted for as the farmlands abandoned decades ago and forested now but still registered as farmland. As a matter of fact, more and more agricultural lands have been abandoned and reclaimed by forests as the primary industry of the nation dwindled and isolated communities in remote areas were evacuated over the past decades. Some of them have been artificially reforested, but the majority seems to have been just left aside to natural reforestation. What makes the matter worse in this prefecture is the rapidly collapsing orange farming. The abandoned orchards turn more easily into bush to make the government statistics hard to catch up.

The farmlands supposedly reclaimed by forests not only resolve the discrepancies among the three estimates in "Agricultural" land area but also ease those in "Forest" area. 
Consider the laser estimate of $81.7 \%$ and the national figure of $70.5 \%$ for "Forest", and leave aside the prefectural figure of $49.5 \%$, which is heavily distorted by the stretched measure explained earlier. By discounting the reforested farmland, the laser estimate of "Forest" area reduces to $78.5 \%$, easing the overall discrepancy. The remaining imbalance of $7.7 \%$ can be explained as an underestimation in the national statistics, the forest area of which is cited from the National Forest Inventory (NFI). According to MATsumoto (2003), a verification against actually measured forestland area revealed an average of $6.7 \%$ underestimation in the NFI figures. Involving only 40 or so sub-compartments totaling only 150 ha, the credibility of this result is limited but it makes ends meet almost completely.

On the other hand, the laser estimate is neither free from errors. Two sources of errors are conceivable. One is associated with cover classification along the flight line, and the other with the two-dimensional expansion of the line classification into area by multiplying the distance between the two adjacent flight lines. In this study, the first source of error can be considered negligible since the cover classification was made at every point of altimetry, i.e. at an interval of $17 \mathrm{~cm}$ along the flight line. Thus the only source of error in this study is the distance between the two adjacent flight lines, which happened to be four $\mathrm{km}$. The closer the adjacent flight lines, the less the error would be, but it is difficult to quantify the latter as a function of the former in the present study. Thus, it would be fair to attribute the final discrepancy of $6.7 \%$ equally to the laser estimate and to the national statistics.

The discrepancies in the "Urban" and "Others" areas among the laser, national and prefectural estimates are less serious than in the "Agriculture" and "Forest" areas. The discrepancies in the "Urban" area are negligible at 4.8, 4.1 and $4.4 \%$ of the prefectural land area respectively in the laser, national and prefectural statistics. Large discrepancies in the "Others" area may seem serious, but the value of $9.4 \%$ of the prefectural land area in the national statistics can be explained as simply inflated in adjustment of its internal imbalance to the authoritative figure of the GSI, while the over-inflated figure of $30.7 \%$ in the prefectural statistics is simply a compensation for the reduction in "Forest" area due to the stretched measure.

\section{CONCLUSION}

Two conclusions can be drawn from the result above. Firstly, the airborne laser profiling proved to be a prospective method of consistent and objective land-cover classification. With its cover classification along the flight line as precise as $17 \mathrm{~cm}$, its overall accuracy depends on the distance between two adjacent flight lines. With only $0.4 \%$ discrepancy with the most authoritative GSI figure on the total land area of the prefecture, the present spacing of $4 \mathrm{~km}$ between the flight lines should be satisfactory enough for the areas as large as a prefecture, but finer spacing would improve the accuracy of land-cover statistics since each cover category occupies only a part of the prefecture.

Secondly, our land-cover classification by airborne laser profiling revealed some of the problems in the existing government land-use statistics primarily based on legal land registration and aerial photography. It also helped to ensure the credibility of the existing land-use statistics by identifying quantitatively the causes and sources of discrepancies between them as well as with the laser-based classification. Coordinated use of the airborne laser altimetry with the existing statistics would certainly improve the overall quality of land-use/landcover statistics of the prefecture and the nation. The most urgent and important task for such coordination is establishment of common definition and terminology.

\section{LITERATURE CITED}

Ehime Prefecture Statistical Office, (2005): Statistical yearbook of Ehime prefecture. Ehime statistical assoc., Matsuyama, VI + 370pp (in Japanese)

Geographical Survey Instrtute, (2006): National municipality aerial statistics. http://www.gsi.go.jp/KOKUJYOHO/MENCHO/200510/ ichiran.htm (in Japanese)

Intergovernmental PANel on Climate Change, (2003): Good practice guidance for land use, land-use change and forestry. Institute for Global Environmental Strategies (IGES) for the IPCC, Hayama, $\mathrm{V}+588 \mathrm{pp}$

LAND and WATER BUREAU, Ministry of LAND,: Land use control backup system. http://lucky.tochi.mlit.go.jp/whats.html (in Japanese)

Matsumoto, M., (2003): Forest information to meet the demands of the Kyoto protocol and Marrakech accords. Proc. Forest Planning Soc.407: 15-24 (in Japanese)

Nelson, R., Short, A. and VAlenti, M., (2004): Measuring biomass and carbon in Delaware using an airborne profiling LiDAR. Scandinavian Journal of Forest Research 19(6): 500-511

(Received 17 January 2007) (Accepted 20 October 2007) 